\title{
Silicon nanowire reinforced by single-walled carbon nanotube and its applications to anti-pulverization electrode in lithium ion battery
}

\author{
Jin-Liang Zang, Ya-Pu Zhao* \\ State Key Laboratory of Nonlinear Mechanics (LNM), Institute of Mechanics, Chinese Academy of Sciences, Beijing 100190, China
}

\section{A R T I C L E I N F O}

\section{Article history:}

Available online 28 April 2011

\section{Keywords:}

A. Nano-structures

A. Lithium ion battery

B. Fragmentation

C. Computational modeling

\begin{abstract}
A B S T R A C T
Lithium ( $\mathrm{Li}$ ) ion batteries are in high demand by most of portable electronic devices. Silicon (Si), owing to its large theoretical energy density $\left(4200 \mathrm{~mA} \mathrm{~h} \mathrm{~g}^{-1}\right)$, is a promising candidate for anode material. However, loss of electrical contact caused by the pulverization that comes from the enormous volume changes during charging and discharging process hinders its application. The aim of the current work is to elucidate the possibility of using carbon nanotubes (CNTs) for the Si reinforcement to minimize the volume expansions. Firstly, $a b$ initio simulations demonstrate that the Li ions can permeate CNT through the tube end freely under the normal charge voltage $(\sim 3.6 \mathrm{~V})$. Then, molecular dynamics simulations are adopted to analyze the strength improvement of silicon nanowires (SiNWs) encapsulated by CNT. The compression simulations of SiNW were performed to reveal the expansion processes during Li ion insertion into the electrode. The results show that the smaller the SiNW is, the better the reinforcing effect is. A phase transition appears in the SiNW encapsulated by CNT during compression, which makes SiNW more ductile. The simulation results provide an effective way for the SiNW reinforcement in the Li ion battery.
\end{abstract}

(c) 2011 Elsevier Ltd. All rights reserved.

\section{Introduction}

With the development of advanced energy storage technologies, investigation of high-power and long-life batteries becomes more and more important. Rechargeable lithium (Li) ion batteries, which have lots of advantages such as high energy density, low cost and environment-friendly, are the most successful energy storage devices and the most suitable energy carriers for portable electronic-equipments. The three most important functional parts of Li ion batteries are the anode, cathode and electrolyte. Lots of materials can be used for these three parts. At present, the most widely used anode material is graphite, whose capacity is $372 \mathrm{~mA} \mathrm{~h} \mathrm{~g}^{-1}$. The low capacity of graphite fails to meet the requirements for more applications. Significant higher capacities are obtained by using antimony (660 $\left.\mathrm{mA} \mathrm{h} \mathrm{g}^{-1}\right)$ [1], tin (994 $\mathrm{mA} \mathrm{h} \mathrm{g}^{-1}$ ) [2]. Among all these materials, the energy density of $\mathrm{Si}$ is the highest (about $4200 \mathrm{~mA} \mathrm{~h} \mathrm{~g}^{-1}$ ) [3], which is one order of magnitude higher than that of graphite. However, the use of $\mathrm{Si}$ as anode material is frustrated by the poor capacity retention and irreversible crystalline structure change. During the charging and discharging process, the $\mathrm{Li}$ ions move from one electrode through the electrolyte to the other. During the formation of Si-Li alloys, the Si crystal structure changes and a maximum volume expansion of $<400 \%$ can occur after complete lithiation $\left(\mathrm{Li}_{22} \mathrm{Si}_{5}\right)$. The electrical contact loss

\footnotetext{
* Corresponding author.

E-mail address: yzhao@imech.ac.cn (Y.-P. Zhao).
}

because of the cracking that results from huge volume changes hinders the commercialization of Si. Significant stress fields are induced when the Li ions keep diffusing in and out of the electrodes. Cracks and fragmentations are gradually formed. After several cycles, the electrode can break into pieces or even powders. Battery failure occurs owing to the loss of electrical contact. Fig. 1, showing the pulverization process, is a transmission electron microscope (TEM) photo of a pristine SiNW with partial Ni coating before and after Li cycling [4].

Therefore, the main issue on the improvement of the Si cycle performance is how to overcome the volume change and to reinforce $\mathrm{Si}$ to prevent the pulverization. Previous studies demonstrated that using nanostructures such as nanofilm, nanowire or nanorod, nanotube and nanoparticle can help Si to accommodate the volume changes. As a result, longer stability in terms of cycle life and less fading in the capacity is expected for the battery $[4,5]$.

Some researchers used different models to interpret that the nanostructures can accommodate the volume expansion well. $\mathrm{Li}$ and Mehl [6] studied the diffusion-induced stresses in several solid microstructures. Chu and Lee [7] investigated the effect of chemical stresses on diffusion and showed that chemical stresses enhance the mass transfer because of increasing diffusivity. Cheng and Verbrugge [8] found that the surface tension effects have much influence on the diffusion-induced stresses if the anode is in nanometer. Aifantis and Dempsey [9] studied crack growth in the nanostructured Li batteries. However, all researches mentioned above mainly focus on the minimizing volume change and pay little 


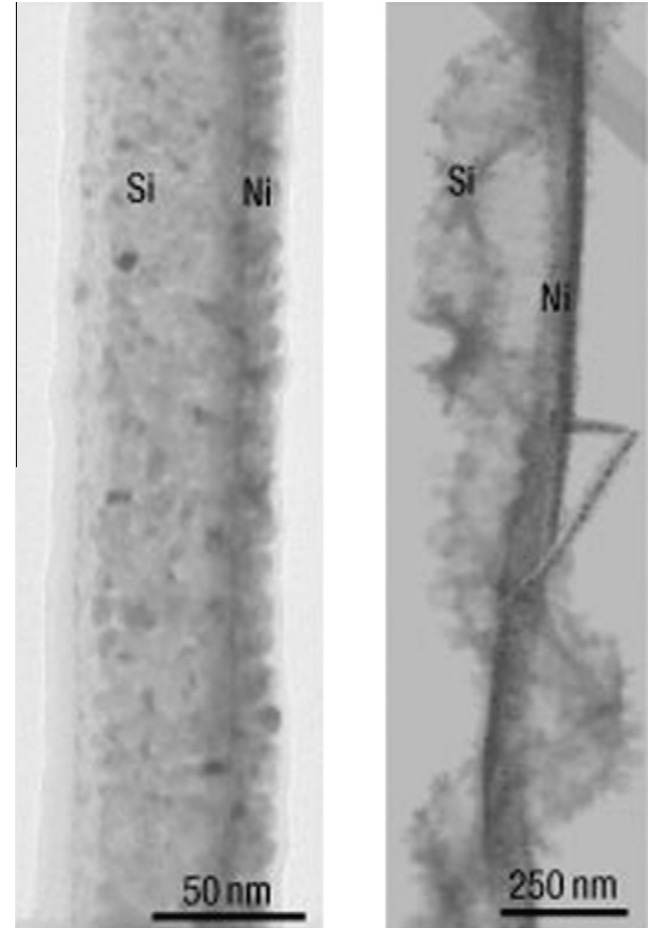

Fig. 1. A pristine SiNW with a partial Ni coating before and after Li cycling [4].

attention on the reinforcement for the Si material. Some groups have used the copper to glue all pulverized Si particles and found that cycling efficiency and rate capability increase [10].

In this study, molecular dynamics (MD) simulations were used to investigate the mechanical behaviors of SiNW encapsulated by CNT. The extremely high Young's modulus and low specific weight make the CNT become the potential reinforcement in the highperformance composites. Zhao group [11] has performed a comparative study of CNT Young's modulus using different methods and predicted that the moduli will be around TPa with different CNT chiralities and diameters. In this work, we use CNT as the reinforcement material and investigate the strength of SiNW encapsulated by CNT for electrode. With the CNT encapsulation, the Li ions firstly permeate the tube through the tube ends; then intercalate into $\mathrm{Si}$ and gradually form the lithiated state. Within the $\mathrm{Li}$ ions intercalation, crystalline $\mathrm{Si}$ becomes amorphous $\mathrm{Li}_{x} \mathrm{Si}$. To reflect the volume expansion of SiNW during the Li ion insertion, a series of compression simulations were performed. The strength of SiNW encapsulated by CNT is much higher than that of a pure SiNW. The phase transition appears in SiNW when it was encased in the CNT, which makes the SiNW more ductile. As a matter of fact, the deformation mechanism of SiNW wrapped by CNT is different from a pure SiNW. Park et al. [12] studied the deformation mechanism and predicted the yield strength of nanowires quantitatively by MD simulations. Gall et al. [13] predicted that the nanowire strength increased with decreasing size. In pure SiNW, the strength is always determined by buckling, shear sliding or dislocation starvation [14] owing to different ratio of length to radius. The phenomenon that smaller is stronger is observed in all simulations. At the moment, it is still difficult to capture the fragmentation process using MD simulations. Some methods like the lattice wave theory of MD [15] will be appreciated to overcome the difficulty of time scale and space scale of the MD.

This paper is organized as follows. Section 2 presents the simulation details about the configurations and parameters. The simulation results and discussions for different SiNWs are given in Section 3. Finally, some concluding remarks are presented in Section 4 .

\section{Simulation method}

MD simulations were performed to investigate the deformation mechanism and strength variation of SiNWs. As shown in Fig. 2, a SiNW with a diameter of $2.0 \mathrm{~nm}$ and a length of $17.0 \mathrm{~nm}$ was constructed. To avoid the end effect of CNT, two CNTs with the length of $37.0 \mathrm{~nm}$ were constructed, which are longer than the inside SiNWs. The two CNTs chiralities are $(18,18)$ and $(31,0)$ and the diameters are $2.45 \mathrm{~nm}$ and $2.43 \mathrm{~nm}$, respectively. To investigate the size effect and length/diameter ratio effect on the deformation, a SiNW with diameter $4.6 \mathrm{~nm}$ and a same SiNW that is encapsulated by a $(36,36)$ CNT were modeled. These two NWs are shown in Fig. 3.

All the MD simulations presented in this work were performed using the large-scale atomic molecular massively parallel simulator (LAMMPS) [16]. The interactions between the Si atoms in the SiNW were simulated using Stillinger-Weber (SW) many-body potential [17] that has been successfully used to trace the fracture and dislocations in plenty of Si nanostructures [18]. The empirical SW interatomic potential consists of two- and three-body interaction terms and was originally fitted to describe the crystalline and liquid Si phases. CNT was parameterized within the consistent valence force field (CVFF) developed by Hagler et al. [19]. The most commonly used potential functional are:

$E=E_{\text {pair }}+E_{\text {bond }}+E_{\text {angle }}+E_{\text {dihedral }}+E_{\text {improper }}$,

where $E_{\text {pair }}$ is van der Waals (vdW) interaction that can be expressed as the Lennard-Jones (LJ) 12-6 function, $E_{\text {pair }}=4 \varepsilon\left[\left(\frac{\sigma}{r}\right)^{12}-\left(\frac{\sigma}{r}\right)^{6}\right], r<r_{c}$. $\sigma$ is the distance where the inter-atom potential is zero, $\varepsilon$ is the depth of the potential well and $r$ is the distance between atoms. $E_{\mathrm{bond}}=K_{b}\left(r-r_{0}\right)^{2}$ is the interaction between pairs of bonded atoms,

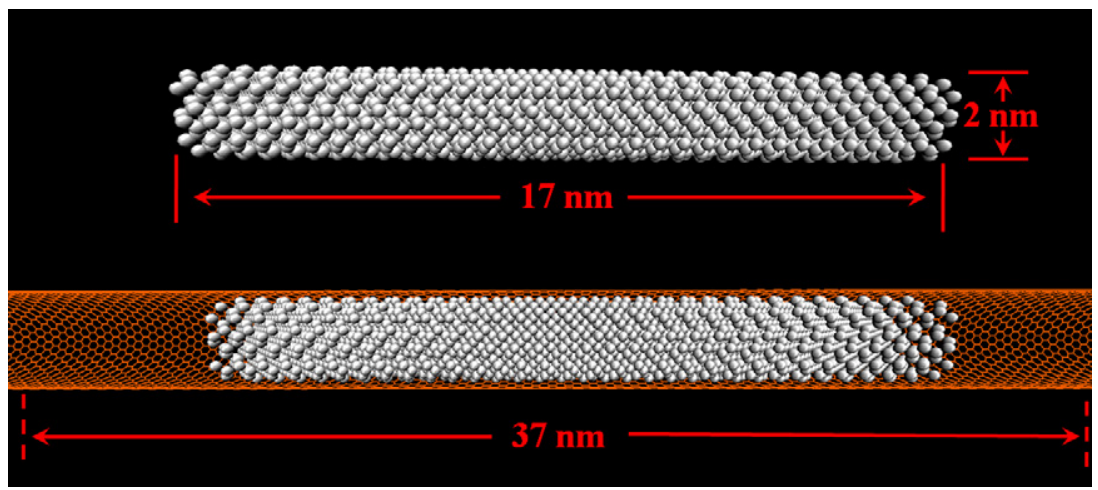

Fig. 2. The configurations for the $2 \mathrm{~nm}$ SiNW and SiNW encapsulated by CNT. 


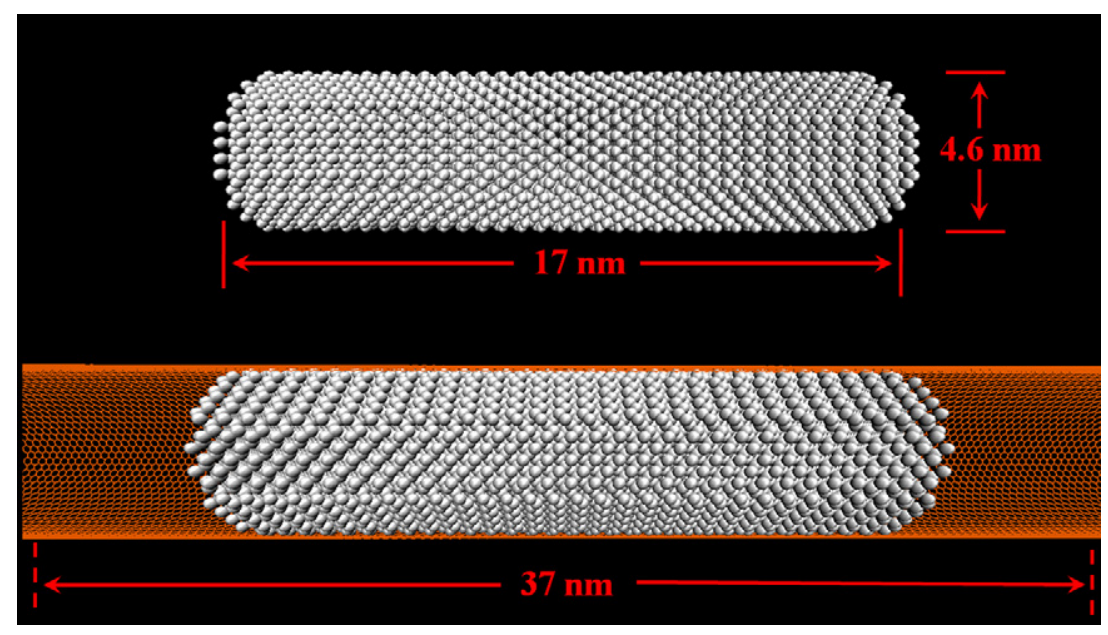

Fig. 3. The configurations for the $4.6 \mathrm{~nm}$ SiNW and SiNW encapsulated by CNT.

$E_{\text {angle }}=K_{a}\left(\theta-\theta_{0}\right)^{2}$ is the interaction of valence angles in the molecule. These two equations represent the harmonic potential that gives the increase in energy as the bond length $r$ deviates from the reference value $r_{0}, \theta$ is the bond angle and the reference value $\theta_{0} . K_{b}, K_{a}$ are the stiffness of the bond and the bond angle, respectively. $E_{\text {dihedral }}=K_{d}\left[1+d_{d} \cos \left(n_{d} \varphi\right)\right], E_{\text {improper }}=K_{i}\left[1+d_{i} \cos \left(n_{i} \varphi\right)\right]$ describe dihedral and improper interactions between quadruplets of atoms. $K_{d}, K_{i}$ are often referred to as the energy barrier height of the dihedral angle and the improper angle. $d_{d}, d_{i}$ whose value is -1 or +1 , represent the direction of the angle. $n_{d}, n_{i}$ are multiplicities. Considering the simulation systems are no-bonding and no-charges, the interactions between the CNT and SiNW are mainly attributed to the nobond vdW interactions. The interactions were represented by $\mathrm{LJ}$ potentials in CVFF forcefield and parameters are $\sigma_{\mathrm{Si}}=4.0534 \AA$, $\sigma_{\mathrm{C}}=3.4745 \AA, \varepsilon_{\mathrm{Si}}=0.0017354 \mathrm{eV}$ and $\varepsilon_{\mathrm{C}}=0.0069383 \mathrm{eV}$, respectively [19]. To get the parameters for the interactions between SiNW and CNT, the Lorentz-Berthelot mixing rule was taken. The cutoff for these interactions was set to be $10.0 \AA$.

Simulations were implemented in the NVT ensemble. The equations of motion were solved using a velocity-Verlet algorithm with a time step of $1.0 \mathrm{fs}$. The system was thermally equilibrated to $300 \mathrm{~K}$ for $0.5 \mathrm{~ns}$ without any loading using a Nose-Hoover thermostat [20]. After the equilibration run, approximation to quasistatic compressive loading in each deformation increment was achieved in two steps, including a $0.2 \mathrm{ps}$ loading process and a 4.8 ps relaxation. The compression speed in the loading process was $0.1 \AA \mathrm{ps}^{-1}$. The loading was applied along the axis of the SiNW, with one end fixed, while the rest of the atoms were displaced in accordance with the other end displacement. The forces at the fixed end were calculated and averaged over a time duration of $5.0 \mathrm{ps}$ and then the stresses were calculated. Periodic boundary conditions were not imposed in any direction. The snapshots of the MD results were processed by the package of VMD [21].

\section{Simulation results and discussions}

In nanostructures, size, shape and surface effects play significant roles in determining the mechanical behaviors of devices. Lots of papers have proved that the Young's modulus, yield stress and fracture toughness $[22,23]$ in the nanoscale are size, shape and surface related. In this part, we will report our simulation results for two kinds of SiNWs with different diameters: $2 \mathrm{~nm}$ and $4.6 \mathrm{~nm}$. The slenderness ratio $\lambda$, the ratio of length to diameter, is used for indicting the size and shape effects on the deformation. In all simulations, the length of SiNW is fixed to $17 \mathrm{~nm}$. Therefore, the slenderness ratios for the two SiNWs are $\lambda_{1}=8.5$ and $\lambda_{2}=3.7$, respectively. The corresponding SiNWs encapsulated by CNTs were also examined. The results show that the strength and ductility of SiNW wrapped by CNT will be improved. In the following part, we will firstly estimate the possible effect of CNT on the Li ions permeability. Then, two different kinds of NWs will be studied separately and a comparative discussion will be presented in the last part of this section.

\subsection{Estimation of CNT effect on Li ions permeation}

In the diffusion process of Li ions, the energy barrier in CNT will be the first obstacle for the Li ions. Fig. 4 shows some kinds of possibilities for the Li ions to get into the CNT. All possibilities can be classified to two categories: wall-ring diffusion and tube end diffusion. Because of the overlapping of electron cloud, there will be energy barriers in the ring of the CNT and the tube end. For the wall-ring type diffusion, the bond curvature affects the overlapping of electron cloud. For the tube end diffusion, the diameter and chirality of the CNT play important roles in determining the energy barrier. We adopted the first-principle simulation to estimate the energy barriers in different diffusion condition. Simulations were performed in Gaussian [24] at 6-31G* level. The potential energy surfaces were scanned in the diffusion direction.

In wall-ring type diffusion, we estimated the energy barriers of three types of ring with different curvatures: uncurved flat ring (Fig. 4a) and rings in two CNTs with different chiralities (Fig. 4b and c). The barriers of the flat ring, ring in $(2,2)$ CNT and ring in $(3,0)$ CNT are $13.38 \mathrm{eV}, 19.18 \mathrm{eV}$ and $21.00 \mathrm{eV}$, which means that battery charging voltage should not lower than 13.38 V. In Fig. $4 d$, the end diffusion was briefly depicted. With a smaller diameter, like $(2,2)$ CNT which is the smallest CNT, plenty of the electrons are overlapped and there is a larger barrier for the $\mathrm{Li}$ ions to get through. However, the electron overlapping decreases with increasing diameter. Therefore, the larger the diameter is, the lower the energy barrier is. To demonstrate this, four CNTs with different diameters were calculated. The barriers for the $(2,2)$ CNT with diameter $2.71 \AA$, $(3,3)$ CNT with diameter $4.07 \AA$, $(4,4)$ CNT with diameter $5.42 \AA$ and $(7,0) \mathrm{CNT}$ with diameter $5.48 \AA$ are $14.47 \mathrm{eV}$, $2.45 \mathrm{eV}, 2.18 \mathrm{eV}$ and $1.63 \mathrm{eV}$, respectively. The last three energy barriers are already lower than the charge voltage of Li ion battery $(\sim 3.6 \mathrm{eV})$ but the nanotube diameter is still small enough. With the increase in diameter, the energy barrier located at the tube end can be ignored. In short, the Li ions can enter the CNT freely through the tube ends other than the wall-ring under the normal charge 


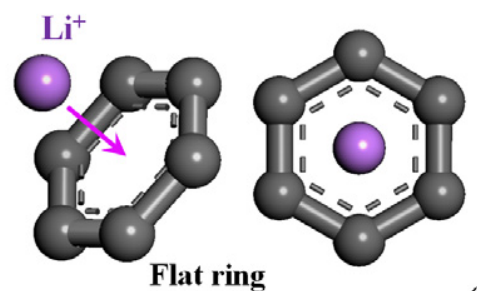

(a) (b)

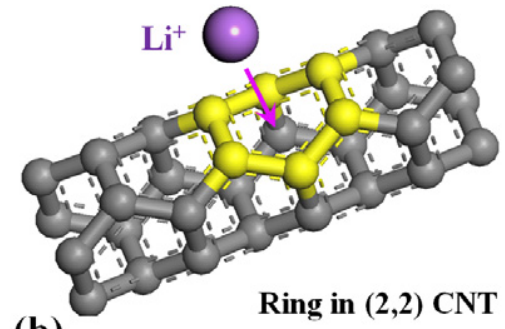

(c)
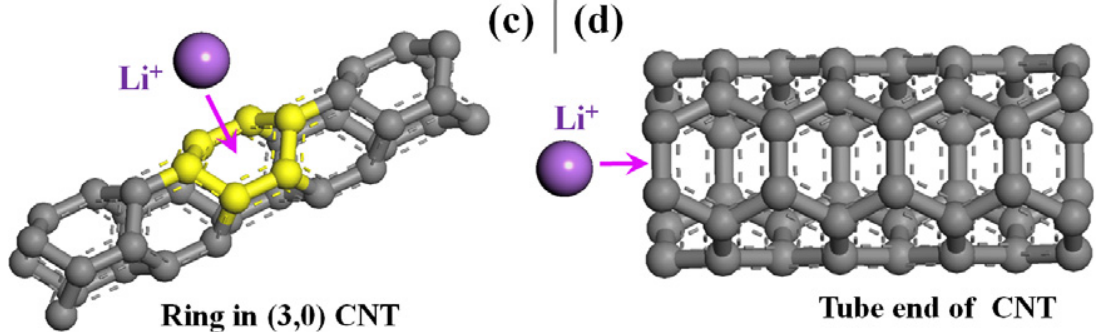

Fig. 4. Possible diffusion ways of Li ion in CNT.

voltage. After the Li ions permeation into the tube, the ions will be stored in the $\mathrm{Si}$, and the CNT will provide a confinement for $\mathrm{Si}$ expansion.

\subsection{SiNW with diameter $2 \mathrm{~nm}$}

Three SiNWs (2 nm pure SiNW, SiNWs encapsulated by $(16,16)$ and $(31,0)$ CNTs), were compressed simultaneously. The forces $F$ at the fixed end were calculated and averaged. Then, the stresses in the SiNW were calculated through the formula $\sigma=F / S$. $S$ is the cross sectional area of the SiNW and $\sigma$ is the stress. The true strain is defined through $\varepsilon=\ln \left(l / l_{0}\right)$. The $l_{0}$ is the initial length of SiNW that is equal to $17.0 \mathrm{~nm}$ and $l$ is the length of SiNW in every deformation step. The true stress-strain curves in these NWs were plotted in Fig. 5. At the very beginning, the three NWs are elastic and the stress-strain curves are the same as each other before the strain exceeds $6 \%$. After that point, the stress-strain curve of the pure SiNW decreases. Actually, $6 \%$ is not the elastic limit for this SiNW. From the Fig. 6, we can clearly get that the crystalline structure was retained and no shear sliding was observed. The failure reason is SiNW buckling due to the larger slenderness ratio. The SiNW cannot bear the load furthermore after the middle part of the SiNW has buckled.

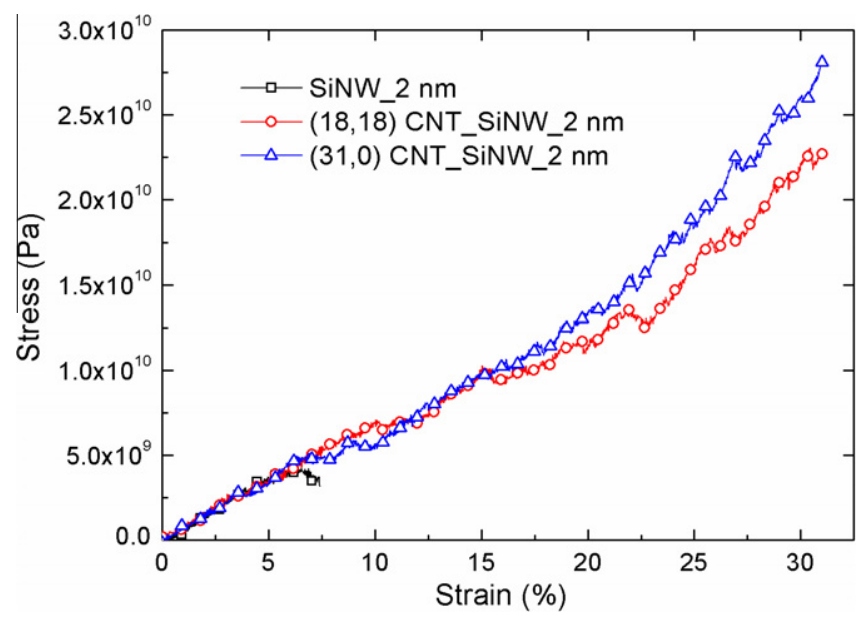

Fig. 5. Representative true stress-strain curves for the $2.0 \mathrm{~nm}$ SiNWs subjected to uniaxial compressive loadings at $300 \mathrm{~K}$.
While the strain achieves 6\%, the SiNWs encapsulated by CNTs are still elastic and can be compressed continuously without buckling. When the strain in the SiNWs reached to $16 \%$, the curves separate. At this moment, the chirality of the CNT will have an effect on the SiNW deformation. The reason is possibly due to bond strength difference of different bond orientation in CNT.

Comparing the pure SiNW with the CNTs encapsulated SiNWs, the stress-strain curves are totally different. Without CNT, the failure of the SiNW is determined by the buckling. With CNT, the CNT can stabilize the SiNW and improve ductibility of SiNW. When we checked atom arrangement evolutions in cross sections of the SiNW, an ordered-disordered-reordered (ODR) process was observed. Fig. 7a shows the initial atoms arrangement. Fig. 7b is the atom configurations snapshot after the $0.5 \mathrm{~ns}$ relaxation at $300 \mathrm{~K}$ and Fig. 7c reveals the final atom arrangements where the co-axial rings can be observed. As a matter of fact, the disordered-reordered process is the key to improve the strength of SiNW. The disordered atom arrangement provides more spaces for the atoms to move in and out and makes the SiNW more ductile. The atoms are reordered along to the CNT. The phase transition occurs in the ODR process.

The structure change of SiNW between the initial configuration and the relaxation one can be attributed to the external intermolecular interactions applied by the CNT. The gap between the internal surface of the CNT and outer surface of SiNW is about $2.25 \AA$. The two surfaces will interact with each other through vdW interactions (see Fig. 8).

According to Refs. [25,26], we can calculate the interactions between two surfaces approximately. Considering the interactions per unit area, the interaction potential can be written as:

$W \approx-\frac{A}{12 \pi D^{2}}\left(1+\frac{D}{R}\right)$

where $D$ is the distance of two surfaces. $A$ is the Hamaker constant that can be represented by $A=\pi^{2} C \rho_{1} \rho_{2}$. $C$ is the interaction constant in the vdW potential. $\rho_{1}$ and $\rho_{2}$ are number densities of two surfaces. $R$ is the radius of the SiNW. Taking the derivative of $W$ with respect to the distance $D$ in Eq. (2) gives the external force per unit area applied by the CNT as:

$F \approx-\frac{\partial W}{\partial D}=-\frac{A}{12 \pi D^{3}}\left(2+\frac{D}{R}\right)$.

For $\mathrm{Si}$ and graphene, the Hamaker constant $A$ is 


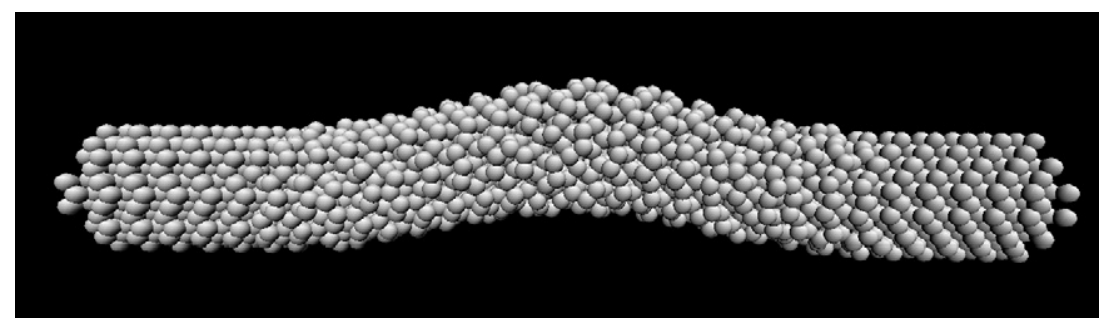

Fig. 6. Structural buckling in SiNW without CNT encapsulation because of the larger slenderness ratio.
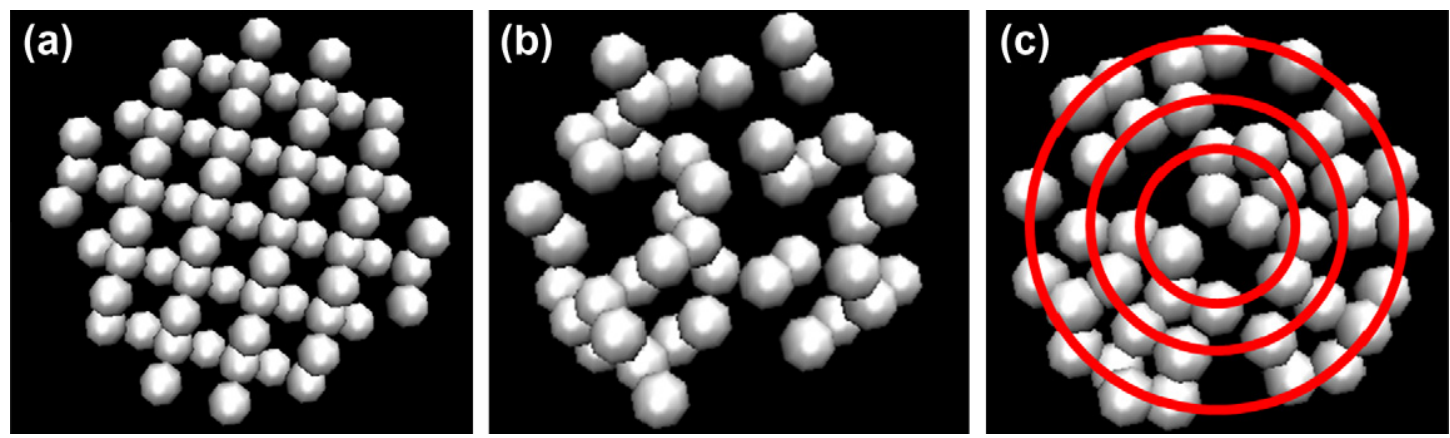

Fig. 7. ODR transition in the CNT encapsulated SiNW.
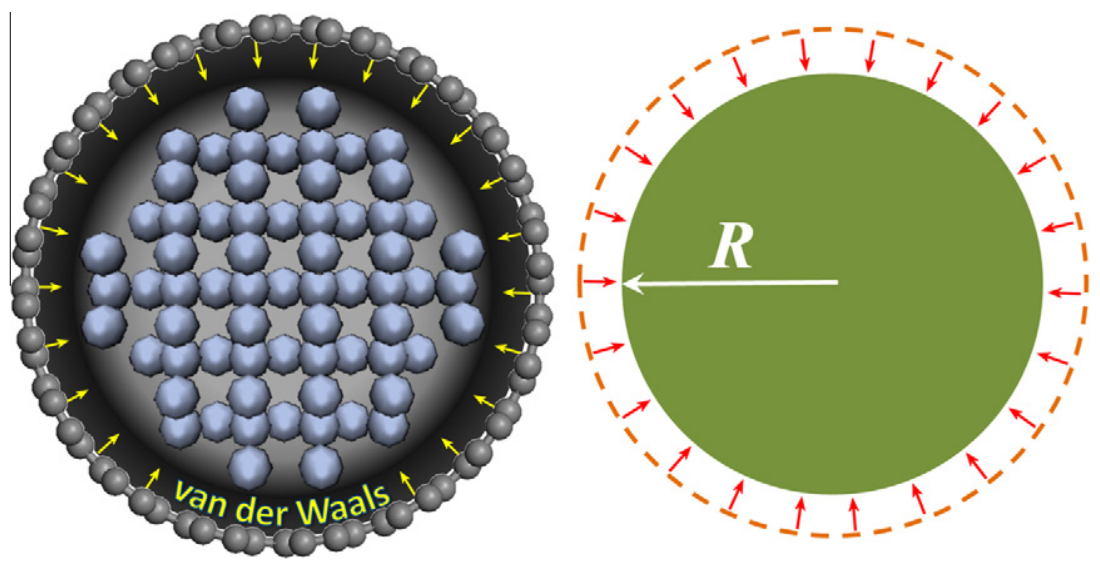

Fig. 8. CNT precompresses the SiNW through the vdW interaction.

$$
\begin{aligned}
A & \approx \pi^{2} C \rho_{\text {Si }} \rho_{\text {graphene }}=\pi^{2} \times 6.3145 \times 5.0095 \times 1.048 \times 10^{-21} \mathrm{~J} \\
& =3.272 \times 10^{-19} \mathrm{~J},
\end{aligned}
$$

where $C=6.3145 \times 10^{-78} \mathrm{~J} \mathrm{~m}^{6}$ is accordance with the simulation parameters. The number densities $\rho_{\mathrm{Si}}, \rho_{\text {graphene }}$ are $5.0095 \times$ $10^{28} \mathrm{~m}^{-3}, 1.048 \times 10^{29} \mathrm{~m}^{-3}$, respectively. Therefore, we can get that the force per unit area is about $1.695 \mathrm{GPa}$. This preloading provides a circle pressure on all radial directions and stabilizes the SiNW in the deformation process.

\subsection{SiNW with diameter $4.6 \mathrm{~nm}$}

To consider the size and shape effects on the deformation of SiNW, the $4.6 \mathrm{~nm}$ SiNWs were simulated using a same loading process. The slenderness ratio of this SiNW is $\lambda_{2}=3.7$. This kind of NW will be more stable and buckling is seldom observed. Fig. 9 gives the stress-strain distributions in the two NWs. As can be seen from the curve of the pure SiNW, there are mainly three drops during the compression process. The insertions show the shear sliding in these three points. However, after the third sliding, atom-losing broke off the simulation process. The yield strength of this SiNW is about $9 \mathrm{GPa}$. The first sliding reduces the effective loading cross sectional area and the following two "yield strengths" cannot restore its initial yield strength of $9 \mathrm{GPa}$ anymore.

The curve in Fig. 9 for the SiWN encapsulated by CNT is quite different from the pure SiNW's. Firstly, the encapsulated SiNW is softer. Comparing the two curves, the slope of the encapsulated one is smaller. The crystalline structure reconstruction of the SiNW with the CNT encapsulation softens the SiNW. Secondly, ODR process also makes the SiNW more ductile. We also estimated that the external pressure applied by the CNT is about $5.31 \mathrm{GPa}$. This is a much higher intermolecular pressure that can change the crystalline structure of the SiNW. Every small reconstruction in the crystalline structure causes the sawtooth-like stress-strain curves. Because of the phase transitions in ODR process, no shear sliding and no drops in the stress-strain curves were observed. 


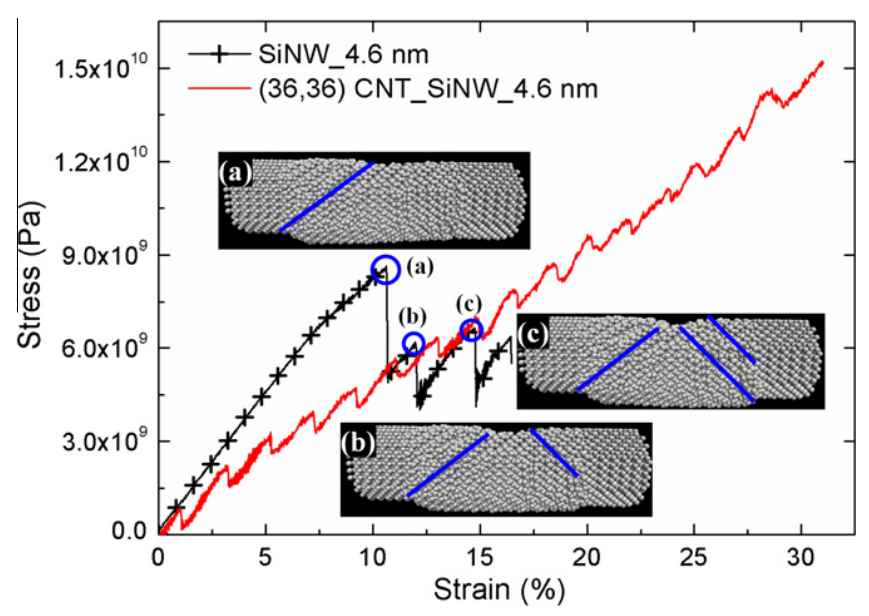

Fig. 9. Stress-strain curves for the $4.6 \mathrm{~nm}$ SiNWs with and without CNT encapsulation.

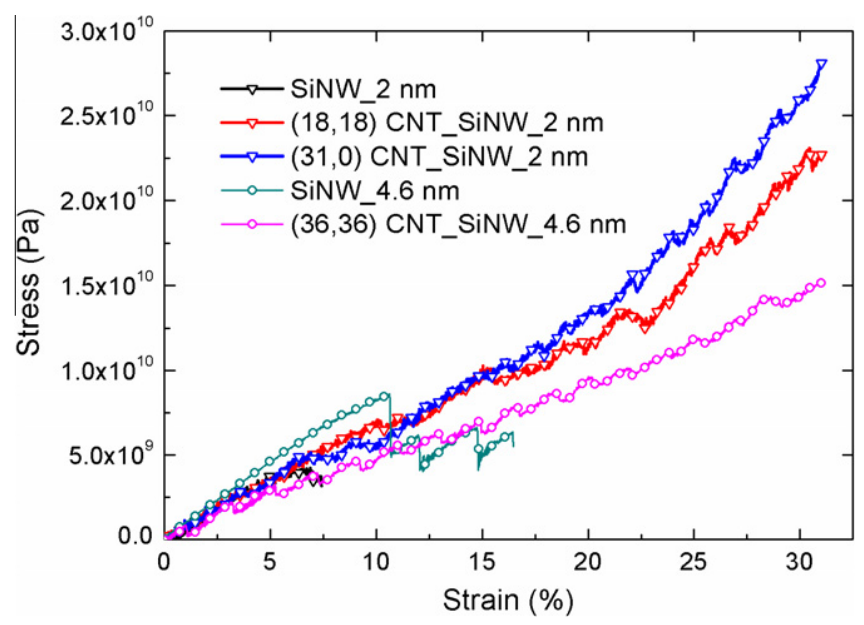

Fig. 10. Comparison of stress-strain curves for different SiNWs.

\subsection{Comparison of two kinds of SiNWs}

We have analyzed two kinds of SiNWs separately and showed the phase transitions in the ODR process. In this section, a comparative study was performed.

We drew all the curves in Fig. 10. The triangle lines represent stress-strain curves related to the SiNWs with $2 \mathrm{~nm}$ diameter and the circle lines are related to the larger diameter ones. In these profiles, we can see that without the CNT, the strength of SiNWs is dominated by the buckling or shear sliding. Accompanying the buckling or shear sliding, the effective loading area decreases and structure failure occurs at low strain levels. With CNT encapsulation, the phase transition appears during the ODR process, this transition provides more spaces for the atoms to move in and out during the compression, and enhances the deformation ability of SiNW. Furthermore, comparing the stress-strain curves of three SiNWs encapsulated by different CNTs, we can find that the slopes of two $2 \mathrm{~nm}$ diameter SiNWs are larger than the slope of $4.6 \mathrm{~nm}$ diameter SiNW. The augmenting moduli of $2 \mathrm{~nm}$ diameter SiNWs are because of their larger surface-to-volume ratios. Meanwhile, it is obvious that the smaller the SiNW is, the better the reinforcing effect is. For instance, when the strain of three SiNWs reaches 31\%, the stresses for $2 \mathrm{~nm}$ diameter SiNWs are 27.99 GPa, 22.70 GPa, and the stress for $4.6 \mathrm{~nm}$ diameter SiNWs is $15.17 \mathrm{GPa}$. The strength decreases from $22.70 \mathrm{GPa}$ to $15.17 \mathrm{GPa}$ when the diameter of SiNW changes from the $4.6 \mathrm{~nm}$ to $2 \mathrm{~nm}$. Higher surface- to-volume ratio of the $2 \mathrm{~nm}$ diameter SiNW makes more surface atoms be reinforced by the CNT.

\section{Conclusions}

In summary, to improve the strength and pulverization resistance ability of $\mathrm{Si}$ anodes in $\mathrm{Li}$ ion batteries, the method that using CNT encapsulation reinforces SiNW is proposed. First, ab initio simulations demonstrate that the CNT will not be an obstacle for the Li ions transportation under the normal charge voltage. Then, we employed MD simulations to study the mechanical behaviors of pure SiNWs and SiNWs with CNTs encapsulation under the compressive loadings at room temperature. Without the CNT, the strength of SiNWs always cannot meet the requirements because of the structural buckling and shear sliding. After the CNT encapsulation, deformation ability and strength of the SiNWs are improved remarkably as a result of phase transition during the ODR process. The phenomenon that smaller is stronger is also observed in the simulations. The results demonstrate that, in contrast to the traditional nanostructured Si electrode, the modulus and the ductibility of SiNW encapsulated by CNT increase with decreasing diameter of SiNW. Therefore, SiNW encapsulated by CNT is a promising nanocomposite for the anode of $\mathrm{Li}$ ion batteries.

\section{Acknowledgements}

This work was jointly supported by the National Natural Science Foundation of China (NSFC, Grant Nos. 60936001, 11021262 and 11011120245), the National Basic Research Program of China (973 Program, Grant No. 2007CB310500).

\section{References}

[1] He XM, Pu WH, Wang L, Ren JG, Jiang CY, Wan CR. Synthesis of nano Sbencapsulated pyrolytic polyacrylonitrile composite for anode material in lithium secondary batteries. Electrochim Acta 2007;52(11):3651-3.

[2] Kwon Y, Kim MG, Kim Y, Lee Y, Cho JP. Effect of capping agents in tin nanoparticles on electrochemical cycling. Electrochem Solid State Lett 2006;9(1):A34-8.

[3] Boukamp BA, Lesh GC, Huggins RA. All-solid lithium electrodes with mixedconductor matrix. J Electrochem Soc 1981;128(4):725-9.

[4] Chan CK, Peng HL, Liu G, Mcllwrath K, Zhang XF, Huggins RA, et al. Highperformance lithium battery anodes using silicon nanowires. Nat Nanotechnol 2008;3(1):31-5.

[5] Song T, Xia JL, Lee JH, Lee DH, Kwon MS, Choi JM, et al. Arrays of sealed silicon nanotubes as anodes for lithium ion batteries. Nano Lett 2010;10(5): 1710-6.

[6] Li JCM, Mehl RF. Physical-chemistry of some microstructural phenomena. Metall Mater Trans A - Phys Metall Mater Sci 1978;9(10):1353-80.

[7] Chu JL, Lee SB. The effect of chemical stresses on diffusion. J Appl Phys 1994;75(6):2823-9.

[8] Cheng YT, Verbrugge MW. Evolution of stress within a spherical insertion electrode particle under potentiostatic and galvanostatic operation. J Power Sources 2009;190(2):453-60.

[9] Aifantis KE, Dempsey JP. Stable crack growth in nanostructured Li-batteries. J Power Sources 2005;143(1-2):203-11.

[10] Sethuraman VA, Kowolik K, Srinivasan V. Increased cycling efficiency and rate capability of copper-coated silicon anodes in lithium-ion batteries. J Power Sources 2011;196(1):393-8.

[11] Zang JL, Yuan QZ, Wang FC, Zhao YP. A comparative study of Young's modulus of single-walled carbon nanotube by CPMD, MD and first principle simulations. Comput Mater Sci 2009;46(3):621-5.

[12] Park HS, Gall K, Zimmerman JA. Shape memory and pseudoelasticity in metal nanowires. Phys Rev Lett 2005;95(25):255504.

[13] Gall K, Diao JK, Dunn ML. The strength of gold nanowires. Nano Lett 2004;4(12):2431-6.

[14] Greer JR, Oliver WC, Nix WD. Size dependence of mechanical properties of gold at the micron scale in the absence of strain gradients. Acta Mater 2005;53(6):1821-30.

[15] Tang QH, Wang TC. Lattice wave theory of molecular dynamics. Sci Sin Phys Mech Astron 2011;41:214-20 (in Chinese).

[16] Plimpton S. Fast parallel algorithms for short-range molecular-dynamics. J Comput Phys 1995;117(1):1-19.

[17] Stillinger FH, Weber TA. Computer simulation of local order in condensed phases of silicon. Phys Rev B 1985;31(8):5262. 
[18] Kang K, Cai W. Brittle and ductile fracture of semiconductor nanowiresmolecular dynamics simulations. Philos Mag 2007;87(14-15): 2169-89.

[19] Hagler AT, Huler E, Lifson S. Energy functions for peptides and proteins. Derivation of a consistent force field including the hydrogen bond from amide crystals. J Am Chem Soc 1974;96(17):5319-27.

[20] Nose S. A unified formulation of the constant temperature moleculardynamics methods. J Chem Phys 1984;81(1):511-9.

[21] Humphrey W, Dalke A, Schulten K. VMD: visual molecular dynamics. J Mol Graph 1996;14(1):33-8.

[22] Guo JG, Zhou LJ, Zhao YP. Size-dependent elastic modulus and fracture toughness of the nanofilm with surface effects. Surf Rev Lett 2008;15(5): 599-603.

[23] Yang ZY, Lu ZX, Zhao YP. Shape effects on the yield stress and deformation of silicon nanowires: a molecular dynamics simulation. J Appl Phys 2009;106(2): 0235.
[24] Frisch MJ, Trucks GW, Schlegel HB, Scuseria GE, Robb MA, Cheeseman JR, Scalmani G, Barone V, Mennucci B, Petersson GA, Nakatsuji H, Caricato M, Li X, Hratchian HP, Izmaylov AF, Bloino J, Zheng G, Sonnenberg JL, Hada M, Ehara M, Toyota K, Fukuda R, Hasegawa J, Ishida M, Nakajima T, Honda Y, Kitao O, Nakai H, Vreven T, Montgomery Jr JA, Peralta JE, Ogliaro F, Bearpark M, Heyd JJ, Brothers E, Kudin KN, Staroverov VN, Kobayashi R, Normand J, Raghavachari K, Rendell A, Burant JC, Iyengar SS, Tomasi J, Cossi M, Rega N, Millam NJ, Klene M, Knox JE, Cross JB, Bakken V, Adamo C, Jaramillo J, Gomperts R, Stratmann RE, Yazyev O, Austin AJ, Cammi R, Pomelli C, Ochterski JW, Martin RL, Morokuma K, Zakrzewski VG, Voth GA, Salvador P, Dannenberg JJ, Dapprich S, Daniels AD, Farkas Ö, Foresman JB, Ortiz JV, Cioslowski J, Fox DJ. Gaussian 09, Revision A.1, Gaussian, Inc., Wallingford CT; 2009.

[25] Israelachvili JN. Intermolecular and surface forces: with the applications to colloidal and biological systems. London: Academic Press; 1985.

[26] Parsegian VA. Van der Waals forces: a handbook for biologists, chemists, engineers, and physicists. New York: Cambridge University Press; 2006. 